(1)

CrossMark

\title{
Demystifying morphomolecular alterations of vasculature in interstitial lung diseases
}

\author{
Toyoshi Yanagihara (1) ${ }^{1,2}$ and Kirk D. Jones ${ }^{3}$
}

Affiliations: ${ }^{1}$ Firestone Institute for Respiratory Health, Research Institute at St Joseph's Healthcare, Dept of Medicine, McMaster University, Hamilton, ON, Canada. ${ }^{2}$ Research Institute for Diseases of the Chest, Graduate School of Medical Sciences, Kyushu University, Fukuoka, Japan. ${ }^{3}$ Dept of Pathology, University of California San Francisco, San Francisco, CA, USA.

Correspondence: Toyoshi Yanagihara, Firestone Institute for Respiratory Health, McMaster University, 50 Charlton Ave East, Hamilton, ON, L8N 4A6 Canada. E-mail: yanagihtamcmaster.ca

@ERSpublications

Focusing on remodelling-associated angiogenesis, both sprouting and intussusceptive, Ackermann and co-workers present histopathology, microvascular anatomy and gene expression in three main subtypes of interstitial lung disease: UIP, NSIP and AFE http://bit.ly/2NtmV6D

Cite this article as: Yanagihara T, Jones KD. Demystifying morphomolecular alterations of vasculature in interstitial lung diseases. Eur Respir J 2020; 55: 1902446 [https://doi.org/10.1183/13993003.02446-2019].

Interstitial lung diseases (ILDs) encompass a complex group of hundreds of lung disorders that affect lung tissue with variable morphologies and clinical presentations. The most extensively studied type of ILD is idiopathic pulmonary fibrosis (IPF), which is characterised by progressive pulmonary fibrosis, a decline in lung function, and high mortality with a histological pattern of usual interstitial pneumonia (UIP). A proportion of patients with other types of ILD also develop a progressive fibrosing phenotype, including idiopathic nonspecific interstitial pneumonia (NSIP), as well as restrictive allograft syndrome (RAS) and idiopathic pleuroparenchymal fibroelastosis (iPPFE) with a histological pattern of alveolar fibroelastosis (AFE). RAS is a novel form of chronic lung allograft dysfunction first described in 2011 [1]. iPPFE was newly designated as a rare entity of idiopathic interstitial pneumonia in 2013 [2]. However, the pathogenesis of both RAS and iPPFE remains largely unknown [1, 3, 4].

These progressive fibrosing ILDs can develop vascular alterations, ultimately leading to pulmonary hypertension (PH) [5]. Microvascular alteration in IPF lungs was first described by EBINA et al. [6], who demonstrated an increased capillary density with increased endothelial proliferation in non-fibrotic lesions, but decreased density in remodelled fibrotic lesions. Vascular remodelling is clinically meaningful, since $\mathrm{PH}$ is considered the single most significant predictor of mortality in IPF patients [7-9] with limited therapeutic options [10]. ЈАСОВ et al. [11] also reported that pulmonary vascular volumes in computed tomography had the strongest correlation with mortality in patients with IPF. Given these facts, it is reasonable to hypothesise that microvascular alterations represent one of the steps in the pathogenesis of pulmonary fibrosis. However, the influence of pulmonary microvascular alterations on the progression of ILD remains poorly understood.

In this issue of the European Respiratory Journal, ACKermann et al. [12] present histopathology, microvascular anatomy and gene expression in three main histopathological subtypes of interstitial lung disease: UIP/IPF; NSIP/iNSIP; and AFE/RAS, iPPFE. This is a comprehensive analysis with state-of-the-art technology focusing on remodelling-associated angiogenesis (figure 1). 


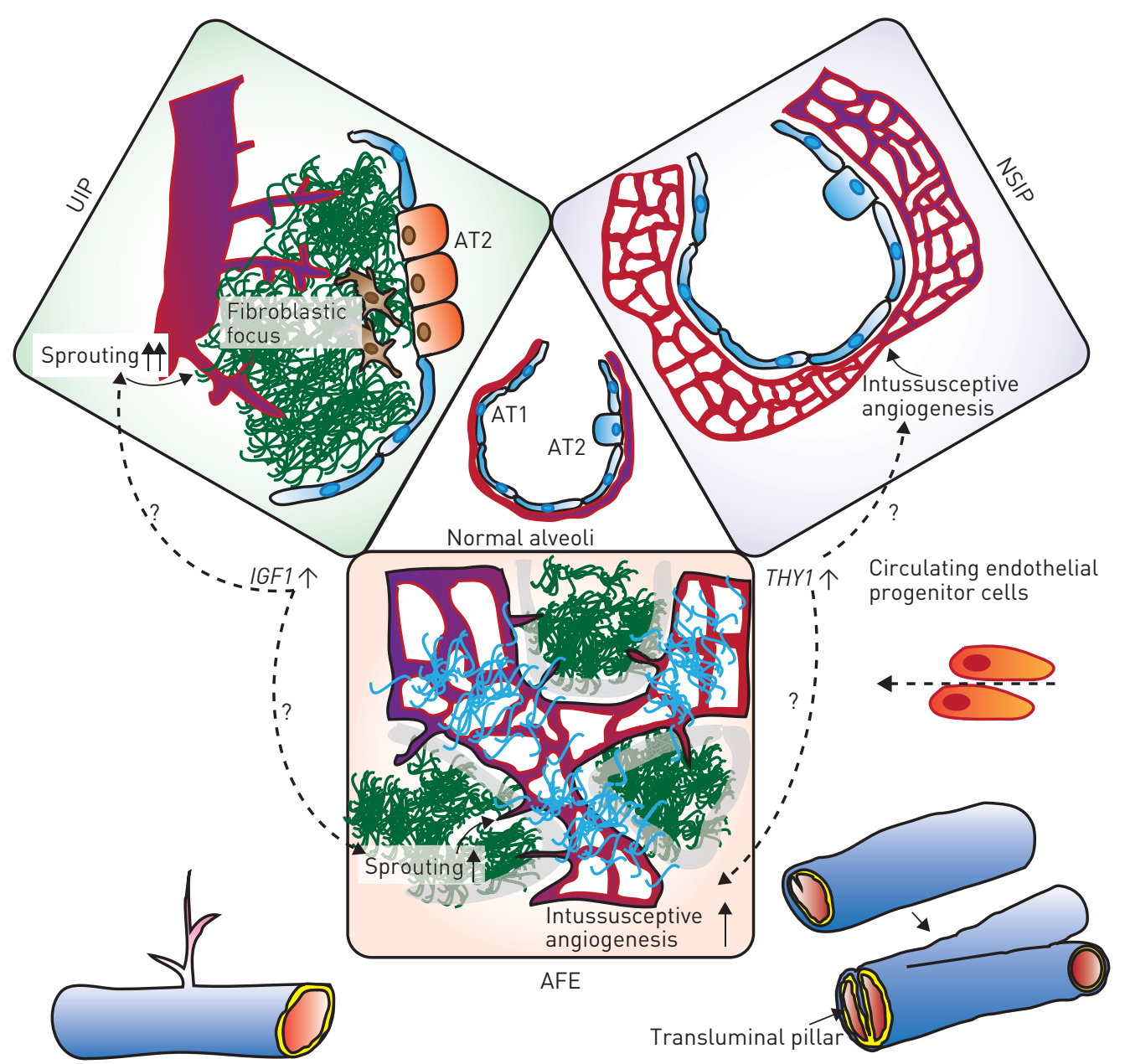

Sprouting angiogenesis

Intussusceptive angiogenesis

FIGURE 1 Schematic illustration of morphomolecular alterations of vasculature in histopathological subtypes of usual interstitial pneumonia (UIP), nonspecific interstitial pneumonia (NSIP) and alveolar fibroelastosis (AFE). There are two types of angiogenesis: sprouting and intussusceptive angiogenesis. Sprouting angiogenesis is characterised by sprouts composed of endothelial cells, which grow toward an angiogenic stimulus. Intussusceptive angiogenesis progresses via initial vasodilation, followed by the formation of a transluminal bridge, the "transluminal pillar" to split an existing blood vessel into two. The expansion of the microvascular network by intussusception is considered to be associated with the recruitment of circulating endothelial progenitor cells. Newly formed sprouting angiogenesis toward fibroblastic foci is frequently found in UIP. Thickened septa composed of dense vascular network expanded by numerous intussusceptive angiogenesis are found in NSIP. Former alveolar air spaces are filled with collagen and increased intussusceptive angiogenesis along with sprouting is found in AFE. IGF1 is upregulated as a common dominator of AFE and UIP, while THY1 is upregulated in NSIP and AFE. AT1: type 1 alveolar epithelial cell; AT2: type 2 alveolar epithelial cell.

The analysis of microvascular corrosion casts of UIP, NSIP and AFE lungs show different morphological patterns. Whereas UIP lungs show a higher density of upstream vascularity and lower density in perifocal blood vessels, NSIP and AFE lungs reveal densely packed alveolar septal blood vessels. Further, neoangiogenesis in UIP is characterised by sprouting of new vessels, while prominent intussusceptive angiogenesis is found in NSIP and AFE. The gene expression analysis using NanoString provides a pivotal insight for differences and similarities of angiogenesis-related gene expression: 16 genes are significantly upregulated in AFE lungs, whereas four genes are differentially expressed in UIP lungs and one gene in NSIP lungs. IGF1 is significantly upregulated as a common denominator of AFE and UIP, whereas MEG3 is significantly expressed in UIP and NSIP. THY1 is highly expressed in NSIP and AFE.

As ACKERMANn et al. [12] described in the study, there are two types of angiogenesis: sprouting angiogenesis and intussusceptive angiogenesis [13]. Sprouting angiogenesis is characterised by a stereotypical series of steps including enzymatic degradation of the capillary basement membrane, endothelial cell proliferation, endothelial cell tube formation, vessel fusion, vessel pruning, and pericyte 
stabilisation [13]. The initial step is driven by hypoxia, which induces angiogenic stimuli, including vascular endothelial growth factor [14]. Given that insulin-like growth factor-1 (IGF-1) is also considered as an angiogenic stimulus for sprouting [15], it is interesting to see IGF1 was upregulated as a common dominator of AFE and UIP [12]. Indeed, ACKERMANN et al. [16] previously showed that inhibition of IGF-1 could prevent sprouting angiogenesis in a tumour model.

On the other hand, intussusceptive angiogenesis progresses via initial vasodilation, followed by the formation of a transluminal bridge, the "transluminal pillar". The expansion of the microvascular network by intussusception is considered to be associated with the recruitment of circulating endothelial progenitor cells [17, 18]. Given the results that a higher frequency of intussusceptive angiogenesis and THY1 upregulation are observed in NSIP and AFE lungs, THY1 might be one of the crucial factors to attract circulating endothelial progenitor cells. Indeed, THY1 is expressed on endothelial cells at sites of chronic inflammation and is involved in the microvascular expansion by intussusceptive neoangiogenesis [19]. It might be informative to evaluate the actual levels of circulating endothelial progenitor cells in these patients in future studies. Lung tissue alteration could impact on the bone marrow, considering the recent report that pulmonary fibrosis caused significant alterations in bone marrow with expansion and activation of monocytic cells via soluble B7H3 and IL-33 upregulation [20].

The study of vascular alteration in interstitial lung diseases by ACKERMANN et al. [12] raises several suggestions for future investigation. First, the study assessed mRNA expression in the explant lungs. The lesion within the explant lungs used for mRNA analysis was not precisely described, but presumably included the fibrotic areas. The results, thus, mainly reflect on angiogenesis in the fibrotic lesions. Vascular degeneration occurs before fibrosis appears in IPF lungs; an analysis of the non-affected areas will be necessary to fully understand the gene expression dynamics regarding vascular alteration, as McDoNOUGH et al. [21] reported recently. Second, a combined analysis of mass spectrometry-based tissue imaging with vessel structure would be informative for unveiling post-translational modification and extracellular matrix shifts in sites with vascular alterations. Third, as investigators discuss in the study [12], the canonical question "Angiogenesis in pulmonary fibrosis: too much or not enough?" raised by HANUMEGOWDA et al. [22] cannot be easily addressed. An analysis of endothelial cell-specific gene expression and biological analysis of isolated endothelial cells from patients in fibrogenesis is warranted to answer this question. Fourth, a preclinical study to target genes identified in the study may be useful to evaluate the therapeutic potential.

In conclusion, the outstanding work performed by ACKERMANn et al. [12] is already contributing to a better understanding of the pathogenesis of fibrosing pulmonary disease, and has opened the door for further studies that will expand our knowledge about the multiple changes in the vasculature and altered vascular cell-epithelial/mesenchymal cell interactions occurring during the progression of the disease.

Conflict of interest: T. Yanagihara has nothing to disclose. K.D. Jones has nothing to disclose.

\section{References}

1 Sato M, Waddell TK, Wagnetz U, et al. Restrictive allograft syndrome (RAS): a novel form of chronic lung allograft dysfunction. J Hear Lung Transplant 2011; 30: 735-742.

2 Travis WD, Costabel U, Hansell DM, et al. An official American Thoracic Society/European Respiratory Society statement: update of the international multidisciplinary classification of the idiopathic interstitial pneumonias. Am J Respir Crit Care Med 2013; 188: 733-748.

3 Chua F, Desai SR, Nicholson AG, et al. Pleuroparenchymal fibroelastosis: a review of clinical, radiological and pathological characteristics. Ann Am Thorac Soc 2019; 16: 1351-1359.

4 Watanabe K. Pleuroparenchymal fibroelastosis: its clinical characteristics. Curr Respir Med Rev 2014; 9: 229-237.

5 Caminati A, Cassandro R, Harari S. Pulmonary hypertension in chronic interstitial lung diseases. Eur Respir Rev 2013; 22: 292-301.

6 Ebina M, Shimizukawa M, Shibata N, et al. Heterogeneous increase in CD34-positive alveolar capillaries in idiopathic pulmonary fibrosis. Am J Respir Crit Care Med 2004; 169: 1203-1208.

7 Lettieri CJ, Nathan SD, Barnett SD, et al. Prevalence and outcomes of pulmonary arterial hypertension in advanced idiopathic pulmonary fibrosis. Chest 2006; 129: 746-752.

8 Patel NM, Lederer DJ, Borczuk AC, et al. Pulmonary hypertension in idiopathic pulmonary fibrosis. Chest 2007; 132: 998-1006.

9 Behr J, Ryu JH. Pulmonary hypertension in interstitial lung disease. Eur Respir J 2008; 31: 1357-1367.

10 Cottin V, Price LC, Valenzuela C. The unmet medical need of pulmonary hypertension in idiopathic pulmonary fibrosis. Eur Respir J 2018; 51: 1702596.

11 Jacob J, Bartholmai BJ, Rajagopalan S, et al. Mortality prediction in idiopathic pulmonary fibrosis: evaluation of computer-based CT analysis with conventional severity measures. Eur Respir J 2017; 49: 1601011.

12 Ackermann M, Stark H, Neubert L, et al. Morphomolecular motifs of pulmonary neoangiogenesis in interstitial lung diseases. Eur Respir J 2020; 55: 1900933.

13 Adair TH, Montani J-P. Angiogenesis. San Rafael, Morgan \& Claypool Life Sciences, 2010.

14 Risau W. Mechanisms of angiogenesis. Nature 1997; 386: 671-674.

15 Jacobo SMP, Kazlauskas A. Insulin-like growth factor 1 (IGF-1) stabilizes nascent blood vessels. J Biol Chem 2015; 290: 6349-6360. 
16 Ackermann M, Morse BA, Delventhal V, et al. Anti-VEGFR2 and anti-IGF-1R-adnectins inhibit Ewing's sarcoma A673-xenograft growth and normalize tumor vascular architecture. Angiogenesis 2012; 15: 685-695.

17 Chamoto K, Gibney BC, Lee GS, et al. CD34+progenitor to endothelial cell transition in post-pneumonectomy angiogenesis. Am J Respir Cell Mol Biol 2012; 46: 283-289.

18 Chamoto K, Gibney BC, Lee GS, et al. Migration of CD11b+ accessory cells during murine lung regeneration. Stem Cell Res 2013; 10: 267-277.

19 Wnuk M, Hlushchuk R, Tuffin G, et al. The effects of PTK787/ZK222584, an inhibitor of VEGFR and PDGFR $\beta$ pathways, on intussusceptive angiogenesis and glomerular recovery from Thy1.1 nephritis. Am J Pathol 2011; 178: 1899-1912.

20 Nakashima T, Liu T, Hu B, et al. Role of B7H3/IL-33 signaling in pulmonary fibrosis-induced profibrogenic alterations in bone marrow. Am J Respir Crit Care Med 2019; 200: 1032-1044

21 McDonough JE, Ahangari F, Li Q, et al. Transcriptional regulatory model of fibrosis progression in the human lung. JCI Insight 2019; 4: 131597.

22 Hanumegowda C, Farkas L, Kolb M. Angiogenesis in pulmonary fibrosis: too much or not enough? Chest 2012; 142: 200-207. 